\title{
Pigment Genodermatoses Affecting Melanocyte Development and Migration from the Neural Crest: Piebaldism, Waardenburg Syndrome and Cross- McKusick-Breen Syndrome
}

\section{Foteini Chatzinasiou ${ }^{1 *}$, Alexander Stratigos ${ }^{2}$ and Dimitrios Rigopoulos ${ }^{1}$}

${ }^{1}$ Department of Dermatology and Venereology, University Hospital "Andreas Sygros" Hospital, Athens, Greece

${ }^{2}$ Department of Dermatology and Venereology, University Hospital ATTIKON, Athens, Greece

\begin{abstract}
Piebaldism, Waardenburg syndrome and Cross-McKusick-Breen syndrome are rare disorders characterized by congenital skin and hair hypopigmentation. Piebaldism is inherited in an autosomal dominant pattern and Waardenburg syndrome is mostly transmitted in an autosomal dominant manner. These diseases are caused by abnormal migration of melanoblasts from the neuroectoderm into the skin. Cross-McKusick-Breen syndrome is an autosomal recessive disorder in which the epidermal melanocyte numbers are normal, but most melanosomes are in stage II or III and are probably characterized by defective melanosomal transfer. This publication describes the clinical and genetic characteristics of the three selected genodermatoses. Although rare and phenotypically diverse, the study of these diseases has yielded significant knowledge on the genes that regulate the migration of melanocytes and the mechanisms that control skin, hair and eye pigmentation.
\end{abstract}

Keywords: Piebaldism; Waardenburg syndrome; Cross-McKusickBreen syndrome

\begin{abstract}
Abbreviations: CMBS: Cross-Mckusick-Breen Syndrome; EDN3: Endothelin-3; EDNRB: Endothelin B Receptor; HSCR: Hirschsprung Disease; KIT: Receptor Tyrosine Kinase 2; KITLG: KIT Ligand, Stem Cell Factor; MC1R: Melanocortin 1 Receptor; MITF: MicrophthalmiaAssociated Transcription Factor; OCA: Oculocutaneous Albinism; OCHS: Oculocerebral Hypopigmentation Syndrome; PAX-3: Paired Box 3; SNAI2: Nail Family Zinc Finger 2; SOX10: Sex Determining Region Y-Box 10; TYR: Tyrosinase; WS: Waardenburg Syndrome; WS1: Waardenburg Syndrome Type I; WS2 : Waardenburg Syndrome Type II; WS3: Waardenburg Syndrome Type III or KleinWaardenburg Syndrome; WS4: Waardenburg Syndrome Type IV or Shah-Waardenburg Syndrome
\end{abstract}

\section{Introduction}

The pigmentation of the skin is, except in rare pathological instances, the result of three pigments or chromophores: melanin, a brown/black (eumelanin) or red/yellow polymer (pheomelanin) produced by melanocytes; hemoglobin in red blood cells in the superficial vasculature; and dietary carotenoids [1]. Melanin, the most important, is formed from tyrosine, via the action of tyrosinase in the lysosome-related organelles of melanocytes, called melanosomes. Melanocytes are dendritic cells, arising from the neural crest during embryonic development and located in the basal layer of the epidermis. The migration of melanoblasts from the neural crest are controlled by genes, such as $c$-KIT receptor tyrosine kinase and the transcription factors $P A X-3$ and MITF. The melanosomes are transferred from a melanocyte to a group of 36 keratinocytes called the epidermal melanin unit, to which they provide melanin.

Interest in the genetics of human pigmentation is longstanding. Variation in human pigmentary form - of skin, hair, and eyes - is one of the most striking polymorphic human traits [2]. More than 150 genes have now been identified that affect pigmentation of the skin, hair and/or eyes such as TYR, MC1R, OCA. The availability of largescale DNA analysis and genome-wide scans, together with our existing knowledge of the genes involved in pigmentation, have contributed to the interpretation of the mechanism of skin pigmentation [2,3].

Disorders of pigmentation can result from migration abnormalities of melanocytes from the neural crest to the skin during embryogenesis. In addition, impairment of melanosome transfer to the surrounding keratinocytes, an alteration in melanin synthesis and a defective degradation or removal of melanin may lead to abnormal skin pigmentation. Immunologic or toxic mediated destructions of melanocytes can also cause pigmentation abnormalities. Disorders of pigmentation are classified in hypo- or hyperpigmentation which can occur as a genetic or acquired disease. They can manifest locally or diffusely $[4,5]$.

Piebaldism, Waardenburg syndrome and Cross-McKusick-Breen syndrome are rare disorders characterized by congenital skin and hair hypopigmentation. Piebaldism is inherited in an autosomal dominant pattern and Waardenburg syndrome is mostly transmitted in an autosomal dominant manner. These diseases are caused by abnormal migration of melanoblasts from the neuroectoderm into the skin. Cross-McKusick-Breen syndrome is an autosomal recessive disorder in which the epidermal melanocyte numbers are normal, but most melanosomes are in stage II or III and are probably characterized by defective melanosomal transfer.

\section{Piebaldism}

\section{Definition}

Piebaldism is a rare, autosomal dominant disorder. The incidence of piebaldism is estimated to be less than 1:20000. Both males and females are equally affected, and no race is spared [6]. The disorder is characterized by the congenital absence of melanocytes in the affected

*Corresponding author: Foteini Chatzinasiou, Department of Dermatology and Venereology, University Hospital ATTIKON, Rimini 1, 124 62, Athens, Greece, E-mail: foteinichatzinasiou@gmail.com

Received January 23, 2015; Accepted February 17, 2015; Published February 22, 2015

Citation: Chatzinasiou F, Stratigos A, Rigopoulos D (2015) Pigment Genodermatoses Affecting Melanocyte Development and Migration from the Neural Crest: Piebaldism, Waardenburg Syndrome and Cross-McKusick-Breen Syndrome. Pigmentary Disorders 2: 168. doi:10.4172/2376-0427.1000168

Copyright: ( 2015 Chatzinasiou F, et al. This is an open-access article distributed under the terms of the Creative Commons Attribution License, which permits unrestricted use, distribution, and reproduction in any medium, provided the original author and source are credited. 
areas of the hair and skin. This striking phenotype of depigmented patches of skin and hair has been observed throughout history, with the first descriptions dating to early Egyptian, Greek and Roman writings. Generation after generation demonstrated a distinctive predictable familial mark-a white forelock. Families have sometimes been known for this mark of distinction, carrying such surnames as Whitlock, Horlick, and Blaylock. The word piebald has been attributed to a combination of the "pie" in the magpie (a bird of black and white plumage) and the "bald" of the bald eagle (Haliaeetus leucocephalus) (which has a white feathered head) [7].

\section{Clinical features}

The lesions are present at birth. The depigmented lesions are static and typically occur on the anterior and posterior trunk, mid-upper arm to wrist, mid-thigh to mid-calf, and shins. A characteristic feature of piebaldism is the presence of hyperpigmented macules within the areas lacking pigmentation and also on normally pigmented skin (Figure 1). The depigmented lesions may repigment spontaneously, or after injury. The presence of lesions since birth, the stability of lesions, and the presence of pigmented patches within lesions differentiate piebaldism from vitiligo. The white forelock is a triangular or diamondshaped midline white macule on the frontal scalp or forehead (Figure 2). Scalp poliosis (white forelock) is present in $80 \%$ to $90 \%$ of patients. All the hairs of the forelock are white and the underlying skin is also amelanotic. Poliosis of the eyebrows and eyelashes is common $[8,9]$. Melanocytes are absent or considerably reduced in depigmented patches histologically and ultrastructurally. They are normal in number in the normally pigmented areas.

\section{Genetics/pathogenesis}

Piebaldism is caused by mutations in the KIT gene (receptor tyrosine kinase). This gene, located on chromosome 4q12, is involved in the differentiation and migration of melanoblasts from the neural crest during the embryonic life. The receptor KIT is a member of the type III group of transmembrane receptor tyrosine kinase and is composed of an amino-terminal extracellular ligand-binding domain, a single transmembrane domain, and a cytoplasmic region. The binding of KITLG (KIT ligand, stem cell factor) to the extracellular domain leads to receptor dimerization, intracellular autophosphorylation and tyrosine kinase activation. The binding of KITLG to KIT regulates the migration of melanocytes, cell proliferation, differentiation, survival,

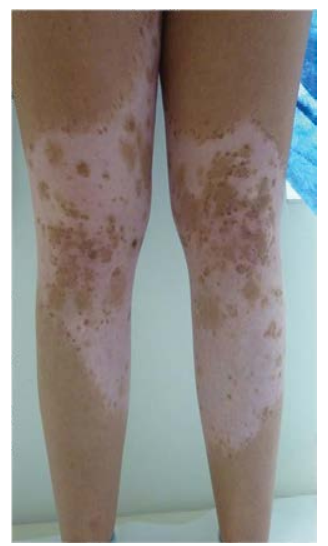

Figure 1: Dermatological lesions in Piebaldism. A 20-year old girl with characteristic features of piebaldism: pigmented dots at the margins or within the white macules on the bilateral legs. These figures are published with the permission of Professor Alexander Stratigos.

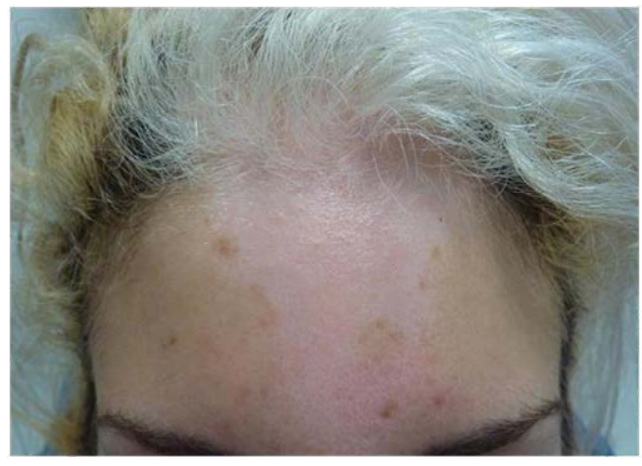

Figure 2: Dermatological lesions in Piebaldism. A 20-year old girl with characteristic features of piebaldism: depigmented macule (leukoderma) on the mid-forehead and white hair (poliosis) on the frontal scalp. These figures are published with the permission of Professor Alexander Stratigos.

melanogenesis and melanosome transfer [10].

Clinical manifestations and phenotypic severity of piebaldism strongly correlate with the site of the mutation within the KIT gene. Dominant negative missense mutations of the intracellular tyrosine kinase domain appear to yield the most severe phenotypes, while mutations in the amino terminal extracellular ligand-binding domain result in haploinsufficiency and are associated with the mildest forms of piebaldism. Intermediate phenotypes are seen with mutations near the transmembrane region. The classic type of static piebaldism is due to $c$-kit gene mutations in the vicinity of codon 620 (Val620Ala, $1859 \mathrm{~T}>\mathrm{C})[6,9]$.

After identification of a missense mutation in the KIT gene in a large family, 32 missense mutations, 17 deletions, 4 insertions, 7 nucleotide splice-site mutations, 2 nonsense mutations and 1 pericentric chromosomal inversion have been identified in the KIT gene or in the chromosomal region of the KIT gene. These genetic studies provide further evidence that the clinical diversity of piebalism depends on the the site and the type of mutation in the KIT gene [10].

\section{Association with other disorders}

Piebaldism may be associated with other disorders. Rare associations have been reported with Hirschprung's disease or aganglionic megacolon, supporting evidence of a network of interacting genes and proteins that regulate melanocytes and the enteric plexus neurons during their development at the time of embryogenesis. Neurofibromatosis Type I has been reportedly associated with piebaldism in a few cases. A piebald patient with congenital dyserythropoietic anemia Type II (HEMPAS) and a patient with Diamond-Blackfan anemia have been also reported. Grover's Disease or transient acantholytic dermatosis limited to the depigmented macules in a patient with piebaldism has been described $[6,9,11]$.

\section{Treatment}

Cosmetic camouflage or skin dyes may be helpful for some patients. Photoprotective preparations should be prescribed to protect the amelanotic areas from burning with sun exposure. The white patches in piebaldism are stable. Surgical treatment with autologous transplantation techniques (minigrafting, suction blister epidermal grafting, grafting of cultured autologous melanocytes, and grafting of noncultured epidermal cell suspensions) can be used to improve the appearance of lesions [8]. Treatment with a combination of dermabrasion and grafting or a combination of Erbium:YAG laser 
Citation: Chatzinasiou F, Stratigos A, Rigopoulos D (2015) Pigment Genodermatoses Affecting Melanocyte Development and Migration from the Neural Crest: Piebaldism, Waardenburg Syndrome and Cross-McKusick-Breen Syndrome. Pigmentary Disorders 2: 168. doi:10.4172/23760427.1000168

Page 3 of 6

surgery for disepithelialization and autologous cultured epidermal grafting on the recipient bed have been reported $[11,12]$

\section{Waardenburg Syndrome (WS)}

\section{Definition}

Waardenburg Syndrome (WS) is a rare auditory-pigmentary disorder caused by physical absence of melanocytes from the skin, hair, eyes, or the stria vascularis of the cochlea. Four WS types have been described on clinical grounds (Table 1).

\section{Clinical features}

On 14 December 1947 the Dutch ophthalmologist and geneticist Waardenburg presented a deaf-mute man with "dystopia punctorum lacrimarum, blepharophimosis and partial iris atrophy" at a meeting of the Dutch Ophthalmological Society. The patient had blue eyes but was bald and Waardenburg did not at the time make the connection between hearing loss, white forelock, unusual eye colour, and dystopia canthorum. He mentioned a report of twins with the same eye abnormality who were "coincidentally" also deaf-mute. Realising that coincidences were multiplying, Waardenburg was prompted to undertake a systematic search among 1050 inmates of five Dutch institutions for the deaf. Waardenburg's results, published in an extensive paper in the American Journal of Human Genetics in 1951, defined the syndrome now named type I Waardenburg syndrome(WS1). He described a new syndrome, consisting of 1 . lateral displacement of the medial canthi and lacrimal points, 2. a hyperplastic, broad, high nasal root, 3. hyperplasia of the medial portions of the eyebrows, 4 . partial or total heterochromia iridum, 5. congenital deafness or partial (unilateral) deafness, and 6. circumscribed albinism of the frontal head hair (white forelock) (Figure 3). Among 840 deaf-mutes in five Dutch institutes for the deaf, 12 cases of the new syndrome were discovered. He characterized the syndrome as autosomal dominant with very high penetrance of dystopia but reduced penetrance of all other features. Waardenburg' estimated the prevalence of his syndrome to be $1 / 42000$ of the population and $1.43 \%$ of the congenitally deaf. Five out of 16 probands having the syndrome were apparently sporadic cases $[13,14]$. Based on their data, Zaman et al., estimated a prevalence of 0.119- 0.208 per 1,000 which was higher than the previously reported prevalence [15]. Swiss ophthalmologist David Klein also made contributions towards the understanding of the syndrome. WS2 was identified in 1971 , to describe cases where "dystopia canthorum" was not present $[12,13]$.

In 1992, the Waardenburg Syndrome Consortium proposed the diagnostic criteria for Waardenburg syndrome type I (WS1). Individuals should be considered to have WS1 if they had two major, or

\begin{tabular}{|c|c|c|}
\hline Type & Clinical features & $\begin{array}{c}\text { Gene mutated } \\
\text { (chromosome) }\end{array}$ \\
\hline I (WS1) & $\begin{array}{c}\text { Dystopia canthorum, broad nasal } \\
\text { root }\end{array}$ & PAX3(2q35) \\
\hline II (WS2) & no dystopia canthorum & $\begin{array}{c}\text { MITF (3p14.2-p14.1) } \\
\text { SNAI2 (SLUG) (8q11) }\end{array}$ \\
\hline $\begin{array}{c}\text { III (WS3) } \\
\text { (Klein-Waardenburg) }\end{array}$ & $\begin{array}{c}\text { Hypoplasia of limb muscles, } \\
\text { contractures of elbows, fingers }\end{array}$ & PAX3 (2q35) \\
\hline $\begin{array}{c}\text { IV (WS4) } \\
\text { (Shah-Waardenburg) }\end{array}$ & Hirschsprung disease & $\begin{array}{c}\text { EDN (20q13.2-q13.3) } \\
\text { EDNRB (13q22) } \\
S O X 10(22 q 13.1)\end{array}$ \\
\hline
\end{tabular}

Table 1: Waardenburg syndrome (WS) has been classified into four main phenotypes, which correspond to mutations of specific genes. Types I and II are the most common forms, while types III and IV are rare. All types of WS typically present with hearing loss and pigmentary abnormalities of the iris, hair, and skin. All of the abnormalities of WS involve the neural crest.

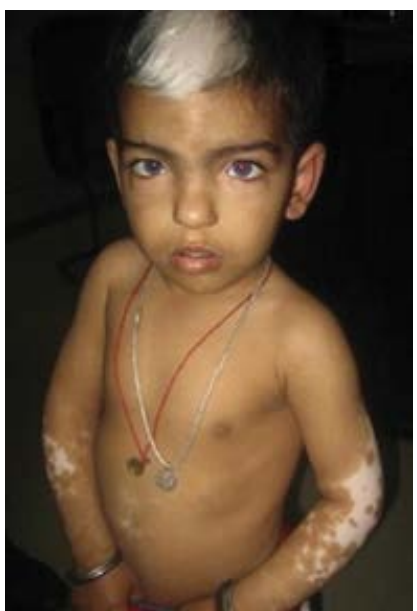

Figure 3: Dermatological lesions in Waardenburg syndrome. A 2-year-old boy with characteristic features of Waardenburg syndrome along with congenital cataract in both eyes: centrally placed white forelock in frontal area, patches of depigmentation on forearm and trunk. Broad nasal bridge with increased intercanthal distance (dystopia canthorum). The child had congenital sensorineural hearing impairment. This figure is published with the permission of the Medical Journal Armed Forces India (MJAFI) [12].

one major and two minor criteria from the list in Table 2 [16]. In 1995, Liu, Newton and Read used the same list to define WS2. Individuals with two major features and who did not have dystopia canthorum were considered to have WS2 [17]. Other types of WS have been identified, but they are less common (Table 1). Type I is characterized by dystopia canthorum, deafness in $60 \%$ of patients, and the distinctive facial features of WS, mainly a high nasal bridge, synophrys, and hypoplasia of the alae nasi (Figure 3). In type II there is no dystopia canthorum and over $80 \%$ of patients have deafness, while $47 \%$ had heterochromia iridum. Type III or Klein-Waardenburg syndrome is a severe form of type I presenting with skeletal abnormalities. Type IV or Shah-Waardenburg syndrome is characterized by the association of WS and Hirschsprung disease [18,19].

\section{Genetics/Pathogenesis}

This syndrome is usually inherited in an autosomal dominant pattern. A small percentage of cases result from new mutations in the gene, these cases occur in people with no history of the disorder in their family. Some cases of type II and type IV WS appear to have an autosomal recessive pattern of inheritance.

Specific genes, including PAX3, MITF, SNAI2, EDNS, EDNRB and SOX10, have been related to WS, with each of these genes playing a significant role in differentiating the numerous manifestations of WS [20] (Table 1).

Types I and III are caused by mutations in the PAX3 (paired box 3 , located on chromosome 2q35) gene. The PAX3 gene belongs to a family of PAX genes that plays a critical role in the formation of tissues and organs during embryonic development. The $P A X$ gene family also encodes a DNA-binding transcription factor expressed in neural crest cells. It plays an important role for the migration and differentiation of melanocytes, which originate from the embryonic neural crest [19]. $P A X 3$ protein is also necessary for the formation of nerve and muscle tissue and certain craniofacial bones. Mutations in PAX3 gene leading to hearing loss and patchy loss of pigmentation such as the limb and facial features in WS type I and II [21].

Type II is caused by mutations in the MITF (microphthalmia- 
Citation: Chatzinasiou F, Stratigos A, Rigopoulos D (2015) Pigment Genodermatoses Affecting Melanocyte Development and Migration from the Neural Crest: Piebaldism, Waardenburg Syndrome and Cross-McKusick-Breen Syndrome. Pigmentary Disorders 2: 168. doi:10.4172/23760427.1000168

Page 4 of 6

\section{Major}

1. Sensorineural deficit hearing threshold greater than $25 \mathrm{~dB}$ for at least two frequencies between 250 and $4,000 \mathrm{~Hz}$, without evidence of middleear effusion and without evidence to suggest that presbyacusis or other factors account for the elevated hearing threshold.

2. Iris pigmentary abnormality:

a. Two eyes of different color.

b. Iris bicolor/segmental heterochromia-an eye with two different colors. The pattern of the different color within the iris usually occurs in a radial segmental distribution and is clearly demarcated.

c. Characteristic brilliant blue (sapphire) iris (alternatively described as "Waardenburg blue eye," sky-blue eyes, or hypopigmented iris). Abnormality of the stroma can be confirmed with slit lamp.

3. Hair hypopigmentation:

a. White forelock: can be present at birth and then disappear later in life, with reappearance in teens or adulthood, or may appear for the first time at any age.

b. Body hair: white hairs within eyebrow, eyelashes, or at other sites on the body.

4. Dystopia canthorum: Lateral displacement of inner canthi, with a reduction of visible sclera medially.

5. First-degree relative previously diagnosed with WS.

Minor

1. Congenital leukoderma -several areas of hypopigmented skin.

2. Synophrys or medical eyebrow flare (a flaring or fanning out of the eyebrow hairs medially, toward the nasal bridge).

3. Broad high nasal root.

a. Evident on full-face view.

b. May or may not occur along with absence of nasofrontal depression evident on profile view (high nasal root).

4. Hypoplasia of alae nasi - hypoplastic lower lateral cartilages, usually resulting in narrow lower third of the nose.

5. Premature graying of the hair-predominance of white scalp hairs before age 30 years; white hairs appearing in the midline rather than at the temples is suggestive of WS.

Table 2: Diagnostic criteria for Waardenburg syndrome (WS) Type I. There are five major and five minor diagnostic criteria for WS (16)

associated transcription factor) gene which is mapped on chromosome 3p14.2-p14.1. MITF is a gene of significant importance for melanocyte development and function. Within melanocytes, MITF also controls production of the pigment melanin. Additionally, MITF regulates the development of specialized cells in the eye called retinal pigment epithelial cells. Mutations in the MITF gene cause a shortage of melanocytes in certain areas of the skin, hair, eyes, and inner ear that leads to hearing loss, pigmentation defects micropthalmia/anopthalmia due to loss of the retinal pigment epithelium [22,23].

The SNAI2 (nail family zinc finger 2) gene (often called SLUG) (located on chromosome $8 \mathrm{q} 11$ ) encodes the snail 2 protein, which plays a role in the development of neural crest cells during embryonic development. Neural crest cells migrate from the developing spinal cord to specific regions in the embryo and give rise to many tissues and cell types such as limb muscles, bones in the face and skull (craniofacial bones), some nerve tissue, and melanocytes. The snail 2 protein probably plays a role in the formation and survival of melanocytes. In some cases of Waardenburg syndrome, type II, both copies of the SNAI2 gene are missing. Lack of snail 2 protein may disrupt the development of melanocytes in certain areas of the skin, hair, eyes, and inner ear, leading to hearing loss and the patchy loss of pigmentation [24].

Type IV is due to either a heterozygous mutation in the SOX10 gene (encoding a transcription factor), or to homozygous mutations in the endothelin-3 (EDN3) or the endothelin B receptor $(E D N R B)$ gene. These mutations impair the ability of melanoblasts to reach their final target sites (inner ear, eye, skin) during embryogenesis [25]. Endothelin Pathway Genes (EDN3, EDNRB) preclude premature differentiation of enteric neuroblasts and influence migration of melanoblasts. EDN3 (endothelin 3, located on chromosome band 20q13.2-q13.3) functions by interacting with the endothelin receptor type $B$ (produced from the $E D N R B$ gene), on the surface of cells. During early development before birth, $E D N 3$ and $E D N R B$ play an important role in neural crest cells. $E D N R B$ (endothelin receptor type $\mathrm{B}$, location on chromosome 13q22). The protein $E D N R B$ is located on the surface of cells and functions as a signaling mechanism, transmitting information from the exterior to the anterior of the cell. The receptor interacts with endothelins to regulate several critical biological processes, including the development and function of blood vessels, the production of certain hormones, and the stimulation of cell growth and division.

Several mutations in the EDN3 and EDNRB genes have been identified in people with WS, type IV (also known as WaardenburgShah syndrome). This type of WS is characterized by changes in skin, hair, and eye coloring; hearing loss and Hirschsprung disease (HSCR), an intestinal disorder that causes severe constipation or intestinal blockage. EDN3 mutations change single nucleotides in the gene, preventing the production of a functional endothelin 3 protein. Because active endothelin 3 is necessary for the formation of enteric nerves and melanocytes, these cell types do not form normally during embryonic development. Missing enteric nerves in certain parts of the intestine cause the signs and symptoms of Hirschsprung disease [26].

SOX10 (sex determining region Y-box 10, which is mapped on chromosome $22 \mathrm{q} 13.1$, is important for melanin synthesis and differentiation of glial cells. During embryonic development, the SOX10 gene is active in neural crest cells. The SOX10 protein directs the activity of other genes (such as MITF) that signal neural crest cells to become more specific cell types. In particular, the SOX10 protein is essential for the formation of nerves in the large intestine (enteric nerves) and melanocytes. At least 15 mutations in the SOX10 gene have been identified in people with Waardenburg syndrome, type IV [27]. Sznajer et al. described a novel SOX10 splice site mutation (c.698$2 \mathrm{~A}>\mathrm{C}$ ) that resulted in type IV WS without Hirschsprung disease [28]. The child presented with vivid blue eye, mental retardation, synophrys, deafness, bilateral complete semicircular canals, and peripheral neuropathy.

\section{Treatment}

Waardenburg syndrome (WS) may be diagnosed at birth or early childhood. There is no specific treatment. The treatment of WS is directed toward the specific symptoms that are apparent in each individual. Early diagnosis and improvement of hearing defects 
Citation: Chatzinasiou F, Stratigos A, Rigopoulos D (2015) Pigment Genodermatoses Affecting Melanocyte Development and Migration from the Neural Crest: Piebaldism, Waardenburg Syndrome and Cross-McKusick-Breen Syndrome. Pigmentary Disorders 2: 168. doi:10.4172/23760427.1000168

Page 5 of 6

are most important for development of children with this disease. Management of the hearing loss associated with WS1 depends on its severity. Cochlear implantation has been successfully utilized in individuals with WS [29]. For those with diminished pigmentation of the irides, lateral displacement of the inner angles of the eyes (dystopia canthorum), and/or other associated ocular abnormalities, ophthalmologists may also recommend certain supportive measures.

\section{Cross-McKusick-Breen Syndrome (CMBS)}

\section{Definition}

This syndrome is known also as oculocerebral hypopigmentation syndrome (OCHS) or Cross syndrome. This is a presumed autosomal recessive disorder based on its familial occurrence and parental consanguinity in some families.

\section{Clinical features}

In 1967, Cross McKusick and Breen reported four siblings in an inbred Amish family, with intellectual disability, microcephaly, neurologic and ocular disorders and hypopigmentation involving skin and hair. These clinical features vary among affected individuals and possibly include hypogonadism as their female patient had undeveloped secondary sexual characteristics at age 12 years and her two younger brothers had cryptorchidism [30-32]. A similar condition was described in 1983 by Preus, Fraser and Wiglesworth in two sisters, both presenting with skin and hair hypopigmentation, dolichocephaly, cataracts, high arched palate, deafness, and severemental retardation (Figure 4). The oldest sister also had growth retardation, small widely spaced teeth, finger contractures, spasticity, hypochromic anemia, myopia, andmoderate hydrocephalus of the lateral ventricles. These authors proposed that their patients had the same condition described by Cross but later in 1987 Patton et al. suggested that Preus and Cross OCHS were distinct entities (Table 3 ) $[31,33,34]$.

CMBS is characterized by white skin (generalized hypopigmentation), blond hair with a yellow-gray metallic sheen. The mixed pattern of hair pigmentation is an important diagnostic sign. One family has been described with two affected siblings and one sibling

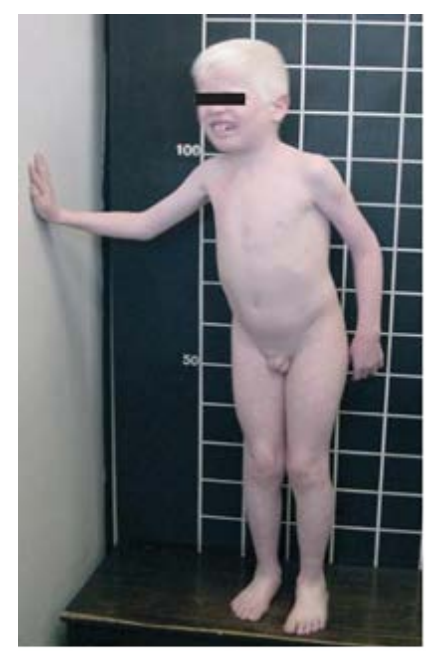

Figure 4: Oculocerebral hypopigmentation syndrome of preus in a 10-yearold boy: generalized cutaneous and hair hypopigmentation, thin build, dolichocephaly, prominent, central upper incisors, spaced teeth and bilateral cryptorchidism. This figure is published with the permission of John Wiley and Sons and Copyright Clearance Center [31].

\begin{tabular}{|c|c|c|}
\hline \multirow{2}{*}{ Clinical findings } & \multicolumn{2}{|c|}{ OCHS Type } \\
\cline { 2 - 3 } & Preus & Cross \\
\hline Generalized hypopigmentation & + & + \\
\hline Growth retardation & $+/-$ & + \\
\hline Dolichocephaly & + & + \\
\hline Cataracts & + & + \\
\hline Microphthalmos & - & + \\
\hline High arched palate & + & - \\
\hline Dental abrnormalities & + & + \\
\hline Congenital sensorineural deafness & + & + \\
\hline Mental deficiency & + & + \\
\hline Athetoid movements & - & + \\
\hline Spasticity & $+/-$ & - \\
\hline Anemia & $+/-$ & + \\
\hline Cryptorchidism & $?$ & + \\
\hline
\end{tabular}

OCHS: Oculocerebral Hypopigmentation Syndrome, +: present, -: absent,?: unknown or undescribed.

Table 3: Main clinical findings of OCHS: Preus and Cross Type Modified version of Table by de Oliveira Sobrinho, et al., [31]

with silver hair who was otherwise unaffected. It has been described in a Gipsy child and in South Africa. Other features reported in the setting of this syndrome include growth retardation, dolichocephaly, small eyes with cloudy corneas, cataracts, jerky nystagmus, high arched palate, small, widely spaced teeth, gingival fibromatosis, severe mental and physical retardation with spastic tetraplegia and athetosis, hypochromic anemia. Occipital cerebral atrophy, coxa valga and generalized osteoporosis have been also described. A reported case with classic findings also had urinary tract abnormality, bilateral inguinal hernia, focal interventricular septal hypertrophy of the heart, vacuolization of myeloid series cells, and distinct ultrastructural features of the skin $[24,34,35]$.

\section{Genetics/pathogenesis}

An interstitial deletion [del(3)(q27.1-1q29)] has been identified in the paternal chromosome of a 4 -year-old female but the molecular defect of this extremely rare disorder is still unknown [30,35].

In the case-report of Patton et al. the electron microscopy confirmed that epidermal melanocyte numbers were normal. The cells also appeared structurally normal and possessed dendrites. There was no evidence of a structural abnormality of the melanosomal matrix. Most melanosomes were in stage II or III with fewer in stage IV. Keratinocytes adjacent to melanocytes were often oedematous and suprabasal cells were vacuolated. The keratinocyte oedema was associated with intercellular granular material, which together might have impeded melanosomal transfer. No autophagic (lysosomal) inclusions or giant melanosomes were observed within melanocytes, keratinocytes, or macrophages [34]. Blood tyrosine levels are normal and the light-coloured hair pigments poorly in tyrosine solution [36]. The generalised hypopigmentation and the mental retardation appear to be linked. Neurones and melanocytes are embryologically derived from the same origin in the neuroectoderm. It appears that melanocytes, or possibly the melanin pigments they produce, are responsible for controlling the functional development of certain neural pathways [34].

\section{Conclusion}

We herein describe the clinical and genetic characteristics of three selected genodermatoses, namely Piebaldism, Waardenburg syndrome and Cross-McKusick- Breen syndrome. The common feature of these disorders is the mutation-based de-arrangements of the normal process 
Citation: Chatzinasiou F, Stratigos A, Rigopoulos D (2015) Pigment Genodermatoses Affecting Melanocyte Development and Migration from the Neural Crest: Piebaldism, Waardenburg Syndrome and Cross-McKusick-Breen Syndrome. Pigmentary Disorders 2: 168. doi:10.4172/23760427.1000168

by which precursors of melanocytes develop at the neural crest and migrate to different peripheral sites. Although rare and phenotypically diverse, the study of these diseases has yielded significant knowledge on the genes that regulate the migration of melanocytes and the mechanisms that control skin, hair and eye pigmentation.

\section{References}

1. Rees JL (2003) Genetics of hair and skin color. Annu Rev Genet 37: 67-90.

2. Rees JL (2011) The genetics of human pigmentary disorders. J Invest Dermatol 131: E12-13.

3. Yamaguchi Y, Hearing VJ (2009) Physiological factors that regulate skin pigmentation. Biofactors 35: 193-199.

4. Fistarol SK, Itin PH (2010) Disorders of pigmentation. J Dtsch Dermatol Ges 8: 187-201.

5. Plensdorf S, Martinez J (2009) Common pigmentation disorders. Am Fam Physician. 2: 109-116.

6. Agarwal S, Ojha A (2012) Piebaldism A brief report and review of the literature. Indian Dermatol Online J 3: 144-147.

7. http://emedicine.medscape.com/

8. Nicolaidou E, Katsambas AD (2014) Pigmentation disorders: hyperpigmentation and hypopigmentation. Clin Dermatol 32:66-72.

9. Thomas I, Kihiczak GG, Fox MD, Janniger CK, Schwartz RA (2004) Piebaldism: an update. Int J Dermatol 43:716-719.

10. Oiso N, Fukai K, Kawada A, Suzuki T (2013) Piebaldism. J Dermatol 40: 330335.

11. López V, Jordá E (2011) Piebaldism in a 2-year-old girl. Dermatol Online J 17: 13

12. Vichare N, Bhargava N (2013) Waardenburg syndrome: A rare case with bilateral congenital cataract: An unusual entity. Med J Armed Forces India 69: 172-174.

13. Waardenburg $P$ (1951) A new syndrome combining developmental anomalies of the eyelids, eyebrows and nose root with pigmentary defects of the iris and head hair and with congenital deafness. Am J Hum Genet 3: 195-253.

14. Read AP, Newton VE (1997) Waardenburg syndrome. J Med Genet 34: 656665.

15. Zaman A, Capper R, Baddoo W (2014) Waardenburg Syndrome: More common than you think! Clin Otolaryngol 40: 44-48.

16. Farrer LA, Grundfast KM, Amos J, Arnos KS, Asher JH Jr, et al. (1992) Waardenburg syndrome (WS) type I is caused by defects at multiple loci, one of which is near ALPP on chromosome 2: first report of the WS consortium. Am J Hum Genet 50: 902-913.

17. Liu XZ, Newton VE, Read AP (1955) Waardenburg syndrome type II: phenotypic findings and diagnostic criteria. Am J Med Genet 55: 95-100.
18. Haddad NM, Ente D, Chouery E, Jalkh N, Mehawej C, et al. (2011) Molecular Study of Three Lebanese and Syrian Patients with Waardenburg Syndrome and Report of Novel Mutations in the EDNRB and MITF Genes. Mol Syndromo 1: 169-175.

19. Wildhardt G, Zirn B, Graul-Neumann LM, Wechtenbruch J, Suckfüll M, et al. (2013) Spectrum of novel mutations found in Waardenburg syndrome types 1 and 2: implications for molecular genetic diagnostics. BMJ Open 3.

20. Jelena B, Christina L, Eric V, Fabiola QR (2014) Phenotypic variability in Waardenburg syndrome resulting from a 22q12.3-q13.1 microdeletion involving SOX10. Am J Med Genet A 164A: 1512-1519.

21. http://ghr.nlm.nih.gov/gene/PAX3

22. http://ghr.nlm.nih.gov/gene/MITF

23. Webb KM, Smith AJ, Dansby LM, Diskin CJ (2014) Waardenburg Syndrome with Familial Unilateral Renal Agenesis: A New Syndrome Variant? Ther Apher Dial [Epub ahead of print]

24. http://ghr.nlm.nih.gov/gene/SNAI2

25. William J, Timothy B, Dirk E (2011) In Andrews' Diseases of the Skin: Clinical Dermatology (11thedtn). Elsevier Saunders, USA.

26. http://ghr.nlm.nih.gov/gene/EDN3

27. http://ghr.nlm.nih.gov/gene/SOX10

28. Sznajer Y, Coldéa C, Meire F, Delpierre I, Sekhara T, et al. (2008) A de novo SOX10 mutation causing severe type 4 Waardenburg syndrome without Hirschsprung disease. Am J Med Genet A 146A: 1038-1041.

29. de Sousa Andrade SM, Monteiro AR, Martins JH, Alves MC, Santos Silva LF, et al. (2012) Cochlear implant rehabilitation outcomes in Waardenburg syndrome children. Int J Pediatr Otorhinolaryngol 76: 1375-1378.

30. Chabchoub E, Cogulu O, Durmaz B, Vermeesch JR, Ozkinay F, et al. (2011) Oculocerebral hypopigmentation syndrome maps to chromosome $3 q 27.1 q 29$. Dermatology 223: 306-310.

31. de Oliveira Sobrinho RP, Steiner CE (2007) What syndrome is this? Oculocerebral hypopigmentation syndrome of preus. Pediatr Dermatol 24: 313-315.

32. Cross HE, McKusick VA, Breen W (1967) A new oculocerebral syndrome with hypopigmentation. J Pediatr 70: 398-406.

33. Preus M, Fraser FC, Wiglesworth FW (1983) An oculocerebral hypopigmentation syndrome. J Genet Hum 31: 323-328.

34. Patton MA, Baraitser M, Heagerty AH, Eady RA (1987) An oculocerebra hypopigmentation syndrome: a case report with clinical, histochemical, and ultrastructural findings. J Med Genet 24: 118-122.

35. Scheinfeld NS (2003) Syndromic albinism: a review of genetics and phenotypes. Dermatol Online J 9: 5.

36. Tony B, Stephen B, Neil C, Christopher G (2010) In Rook's Textbook of Dermatology (8thedtn). Wiley-Blackwell, UK 